\title{
Flight Gate Assignment with a Quantum Annealer
}

Tobias Stollenwerk, Elisabeth Lobe, Martin Jung

German Aerospace Center (DLR)

SIAM Annual Meeting, Portland

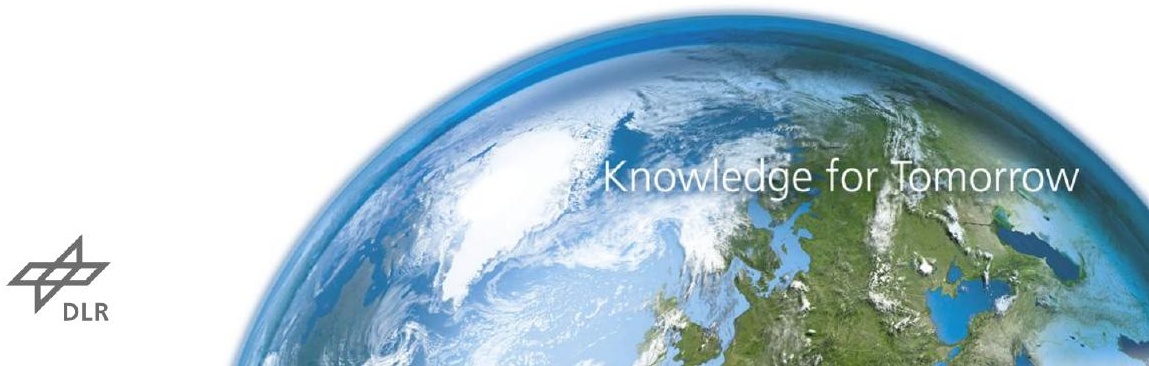




\section{Flight Gate Assignment}

A day at Frankfurt Airport

- about 1300 aircraft movements (arrival and departure)

- more than $90 \%$ are passenger flights

- more than 170000 passengers

- about $60 \%$ transfer passengers

- 278 gates
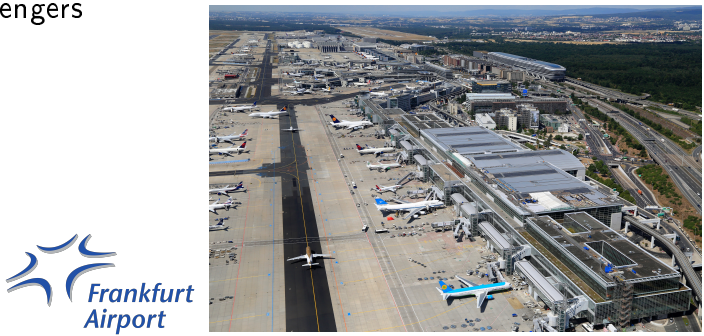
www.DLR.de - Chart $3>$ July 13, $2018>$ T. Stollenwerk > Flight Gate Assignment with a Quantum Annealer

\section{Passenger Flows}

- $F, G$ sets of flights and gates

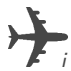

gate $\alpha$

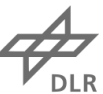


www.DLR.de - Chart $3>$ July 13, $2018>$ T. Stollenwerk > Flight Gate Assignment with a Quantum Annealer

\section{Passenger Flows}

- $F, G$ sets of flights and gates
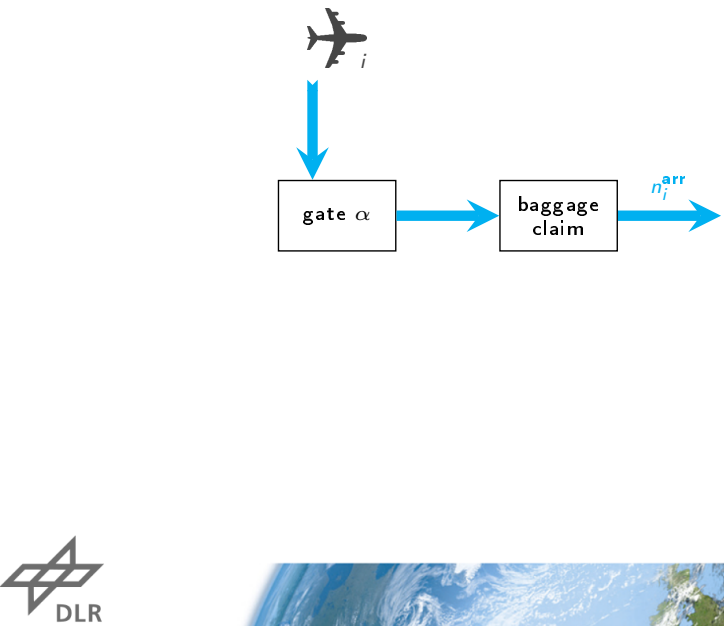


\section{Passenger Flows}

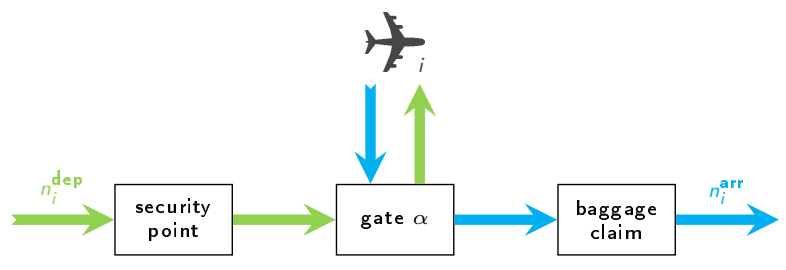

- $F, G$ sets of flights and gates

- $n_{i}^{\text {dep/arr }}$

passengers which depart/ arrive with flight $i$

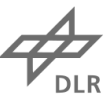




\section{Passenger Flows}

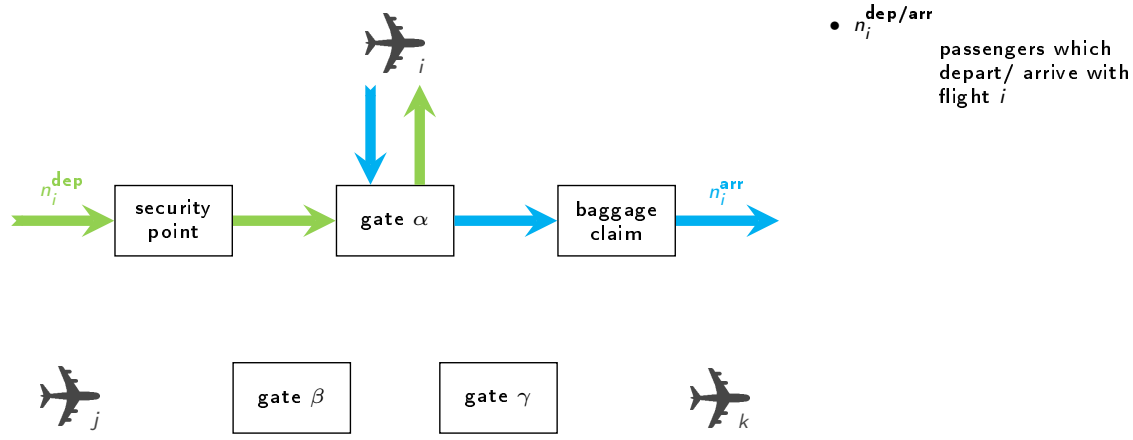

- $F, G$ sets of flights and gates

passengers which depart/ arrive with flight $i$ 


\section{Passenger Flows}

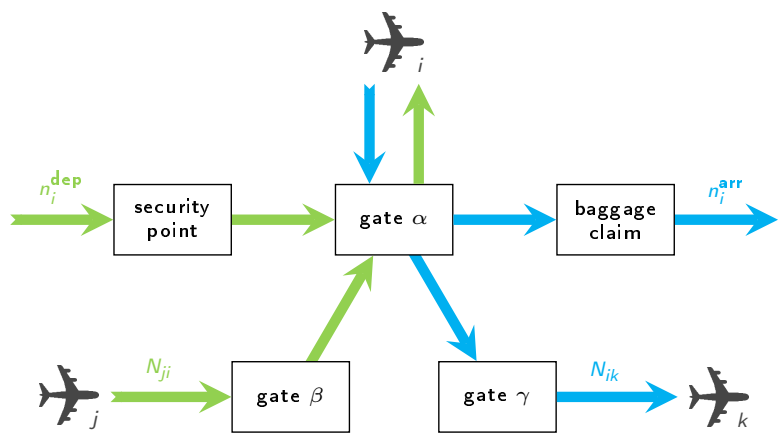

- $F, G$ sets of flights and gates

- $n_{i}^{\text {dep/arr }}$

passengers which depart/ arrive with flight $i$

- $N_{i j} \quad$ transfer passengers from flight $i$ to $j$

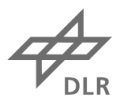




\section{Passenger Flows}

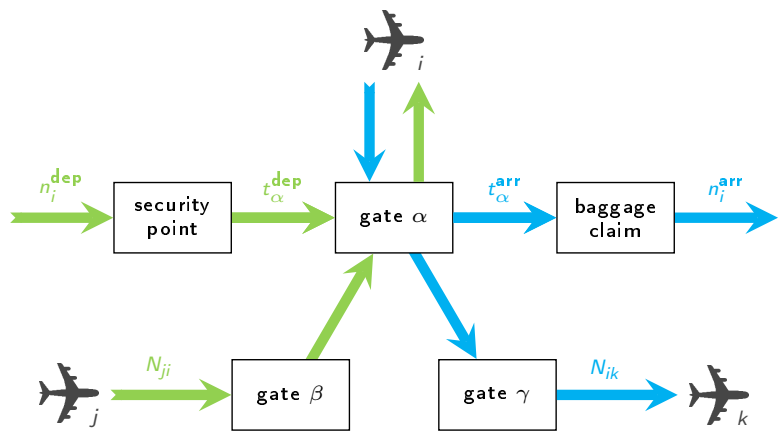

- $F, G$ sets of flights and gates

- $n_{i}^{\text {dep/arr }}$

passengers which depart/ arrive with flight $i$

- $N_{i j} \quad$ transfer passengers from flight $i$ to $j$

- $t_{\alpha}^{\text {dep/arr }}$

average time to arrive at/ leave from gate $\alpha$

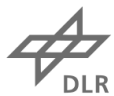




\section{Passenger Flows}

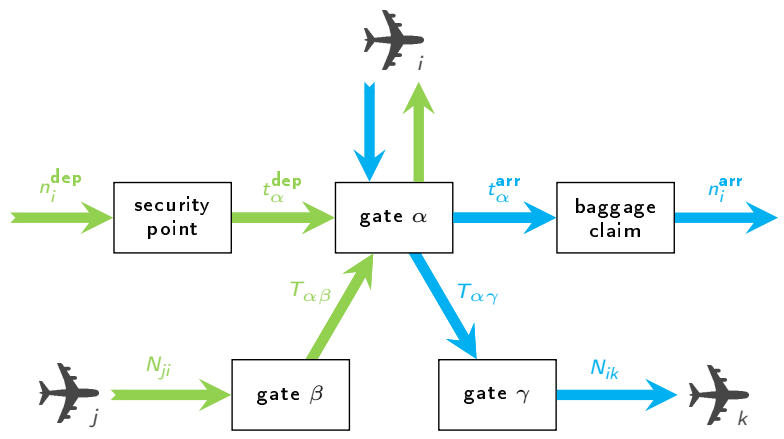

- $F, G$ sets of flights and gates

- $n_{i}^{\text {dep/arr }}$

passengers which depart/ arrive with flight $i$

- $N_{i j} \quad$ transfer passengers from flight $i$ to $j$

- $t_{\alpha}^{\text {dep/arr }}$

average time to arrive at/ leave from gate $\alpha$

- $T_{\alpha \beta}$ average time to get from gate $\alpha$ to $\beta$

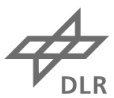




\section{Passenger Flows}

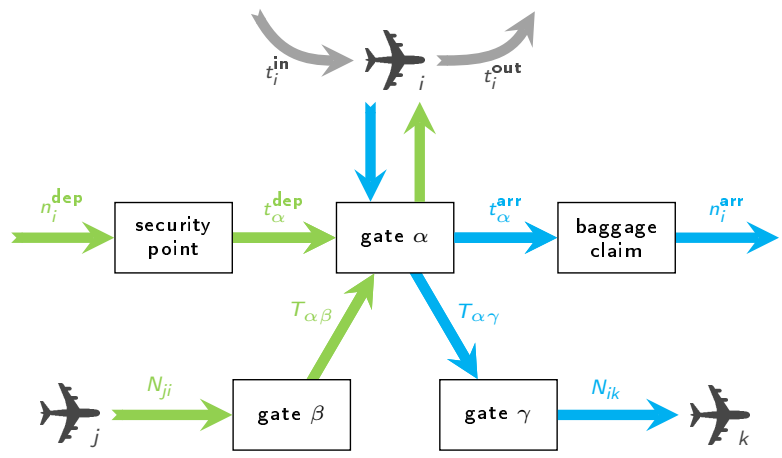

- $F, G$ sets of flights and gates

- $n_{i}^{\text {dep/arr }}$

passengers which depart/ arrive with flight $i$

- $N_{i j} \quad$ transfer passengers from flight $i$ to $j$

- $t_{\alpha}^{\text {dep/arr }}$

average time to arrive at/ leave from gate $\alpha$

- $T_{\alpha \beta}$ average time to get from gate $\alpha$ to $\beta$

- $t_{i}^{\text {in/out }}$ arrival/departure time of flight $i$

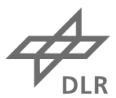




\section{Passenger Flows}

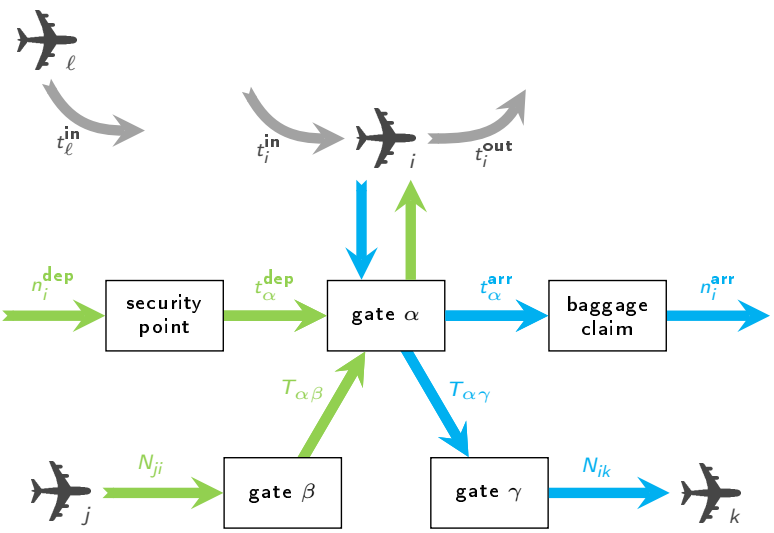

- $F, G$ sets of flights and gates

- $n_{i}^{\text {dep/arr }}$

passengers which depart/ arrive with flight $i$

- $N_{i j} \quad$ transfer passengers from flight $i$ to $j$

- $t_{\alpha}^{\text {dep/arr }}$

average time to arrive at/ leave from gate $\alpha$

- $T_{\alpha \beta}$ average time to get from gate $\alpha$ to $\beta$

- $t_{i}^{\text {in/out }}$ arrival/departure time of flight $i$

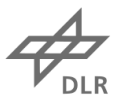




\section{Passenger Flows}

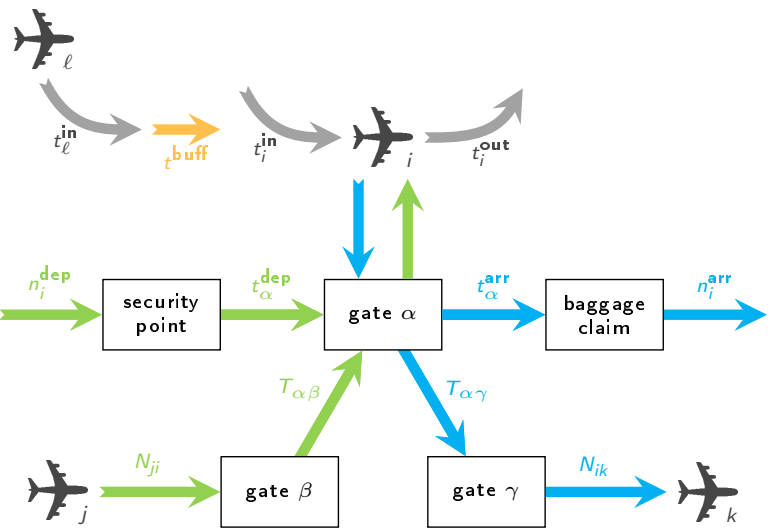

- $F, G$ sets of flights and gates

- $n_{i}^{\text {dep/arr }}$

passengers which depart/ arrive with flight $i$

- $N_{i j} \quad$ transfer passengers from flight $i$ to $j$

- $t_{\alpha}^{\text {dep/arr }}$

average time to arrive at/ leave from gate $\alpha$

- $T_{\alpha \beta}$ average time to get from gate $\alpha$ to $\beta$

- $t_{i}^{\text {in/out }}$ arrival/departure time of flight $i$

- $t^{\text {buff }}$ buffer time between two flights at the same gate

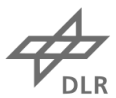




\section{Question}

Which flight should be assigned to which gate, such that the total transit time of the passengers in minimal?

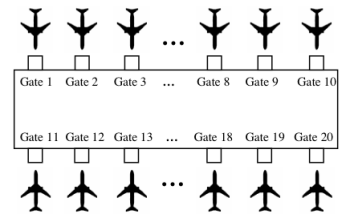




\section{Question}

Which flight should be assigned to which gate, such that the total transit time of the passengers in minimal?

$$
A: F \rightarrow G
$$

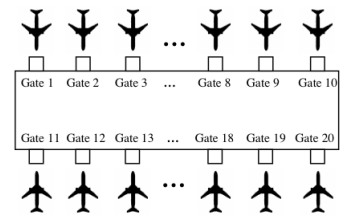




\section{Question}

Which flight should be assigned to which gate, such that the total transit time of the passengers in minimal?

$$
A: F \rightarrow G
$$

$\Rightarrow$ Quadratic Assignment Problem

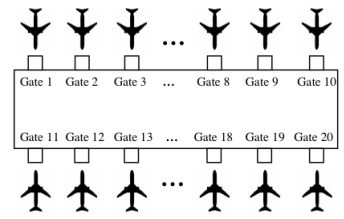




\section{Question}

Which flight should be assigned to which gate, such that the total transit time of the passengers in minimal?

$$
A: F \rightarrow G
$$

$\Rightarrow$ Quadratic Assignment Problem

- fundamental problem in combinatorial optimization

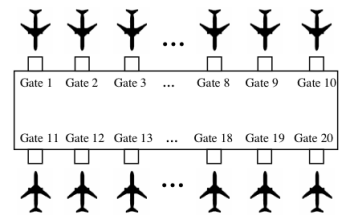




\section{Question}

Which flight should be assigned to which gate, such that the total transit time of the passengers in minimal?

$$
A: F \rightarrow G
$$

$\Rightarrow$ Quadratic Assignment Problem

- fundamental problem in combinatorial optimization

- standard formulation is NP-hard

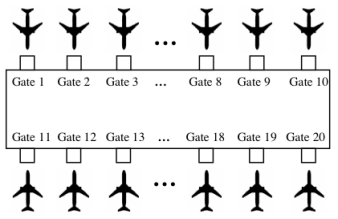




\section{Question}

Which flight should be assigned to which gate, such that the total transit time of the passengers in minimal?

$$
A: F \rightarrow G
$$

$\Rightarrow$ Quadratic Assignment Problem

- fundamental problem in combinatorial optimization

- standard formulation is NP-hard

- seems to exploit possible advantages of the D-Wave machine

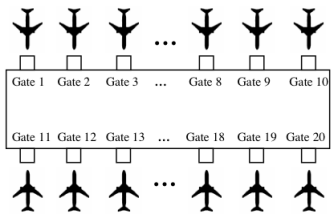




\section{FGA Binary Program}

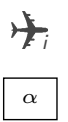

Variables $x \in\{0,1\}^{F \times G}$ with

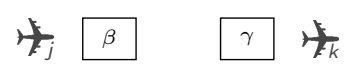

$$
x_{i \alpha}= \begin{cases}1, & \text { if flight } i \text { takes gate } \alpha, \\ 0, & \text { otherwise }\end{cases}
$$




\section{FGA Binary Program}

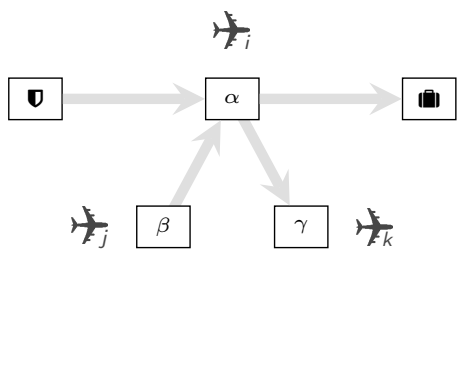

Minimizing the total transfer time with objective function

$$
O(x)
$$




\section{FGA Binary Program}

Variables $x \in\{0,1\}^{F \times G}$ with

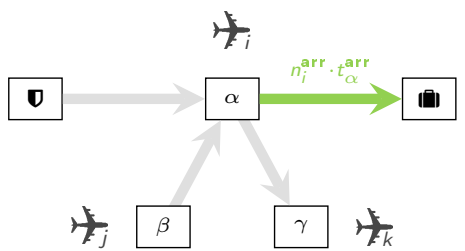

$$
x_{i \alpha}= \begin{cases}1, & \text { if flight } i \text { takes gate } \alpha, \\ 0, & \text { otherwise }\end{cases}
$$

Minimizing the total transfer time with objective function

$$
\begin{aligned}
O(x) & =O_{\text {arr }}(x) \\
& =\sum_{i \alpha} n_{i}^{\text {arr }} t_{\alpha}^{\text {arr }} x_{i \alpha}
\end{aligned}
$$




\section{FGA Binary Program}

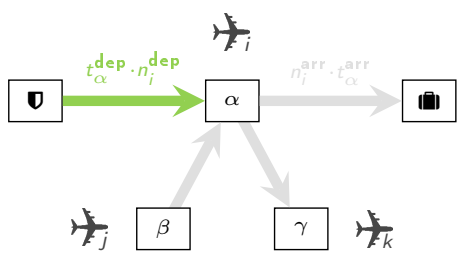

$$
x_{i \alpha}= \begin{cases}1, & \text { if flight } i \text { takes gate } \alpha, \\ 0, & \text { otherwise }\end{cases}
$$

Minimizing the total transfer time with objective function

$$
\begin{aligned}
O(x) & =O_{\text {arr }}(x) \quad+O_{\text {dep }}(x) \\
& =\sum_{i \alpha} n_{i}^{\text {arr }} t_{\alpha}^{\text {arr }} x_{i \alpha}+\sum_{i \alpha} n_{i}^{\text {dep }} t_{\alpha}^{\text {dep }} x_{i \alpha}
\end{aligned}
$$




\section{FGA Binary Program}

Variables $x \in\{0,1\}^{F \times G}$ with

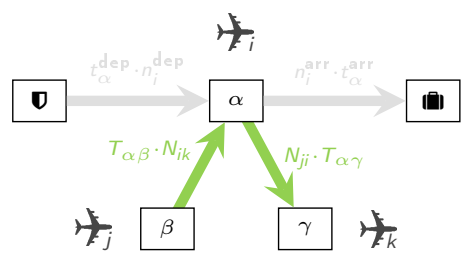

$$
x_{i \alpha}= \begin{cases}1, & \text { if flight } i \text { takes gate } \alpha, \\ 0, & \text { otherwise }\end{cases}
$$

Minimizing the total transfer time with objective function

$$
\begin{aligned}
O(x) & =O_{\text {arr }}(x) \quad+O_{\text {dep }}(x) \quad+O_{\text {transfer }}(x) \\
& =\sum_{i \alpha} n_{i}^{\text {arr }} t_{\alpha}^{\text {arr }} x_{i \alpha}+\sum_{i \alpha} n_{i}^{\text {dep }} t_{\alpha}^{\text {dep }} x_{i \alpha}+\sum_{i j \alpha \beta} N_{i j} T_{\alpha \beta} x_{i \alpha} x_{j \beta}
\end{aligned}
$$




\section{FGA Binary Program}

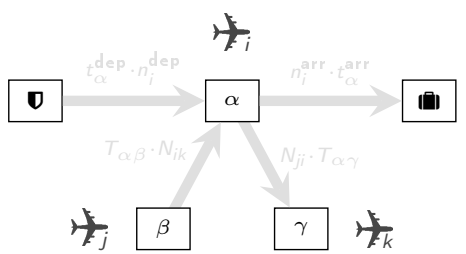

$$
x_{i \alpha}= \begin{cases}1, & \text { if flight } i \text { takes gate } \alpha, \\ 0, & \text { otherwise }\end{cases}
$$

Minimizing the total transfer time with objective function

$$
\begin{aligned}
O(x) & =O_{\text {arr }}(x) \quad+O_{\text {dep }}(x) \quad+O_{\text {transfer }}(x) \\
& =\underbrace{\sum_{i \alpha} n_{i}^{\text {arr }} t_{\alpha}^{\text {arr }} x_{i \alpha}+\sum_{i \alpha} n_{i}^{\text {dep }} t_{\alpha}^{\text {dep }} x_{i \alpha}}_{\text {linear }}+\underbrace{\sum_{i j \alpha \beta} N_{i j} T_{\alpha \beta} x_{i \alpha} x_{j \beta}}_{\text {quadratic }}
\end{aligned}
$$




\section{FGA BP Constraints}

1. One gate per flight

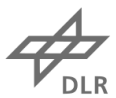




\section{FGA BP Constraints}

1. One gate per flight

$$
\sum_{\alpha} x_{i \alpha}=1 \quad \forall i \in F
$$

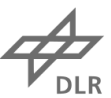




\section{FGA BP Constraints}

1. One gate per flight

$$
\sum_{\alpha} x_{i \alpha}=1 \quad \forall i \in F
$$

2. Different gates if standing times of two flights overlap forbidden pairs 


\section{FGA BP Constraints}

1. One gate per flight

$$
\sum_{\alpha} x_{i \alpha}=1 \quad \forall i \in F
$$

2. Different gates if standing times of two flights overlap forbidden pairs

$$
P=\left\{(i, j) \in F^{2}: t_{i}^{\text {in }}<t_{j}^{\text {in }}<t_{i}^{\text {out }}+t^{\text {buff }}\right\}
$$




\section{FGA BP Constraints}

1. One gate per flight

$$
\sum_{\alpha} x_{i \alpha}=1 \quad \forall i \in F
$$

2. Different gates if standing times of two flights overlap forbidden pairs

$$
\begin{aligned}
& P=\left\{(i, j) \in F^{2}: t_{i}^{\text {in }}<t_{j}^{\text {in }}<t_{i}^{\text {out }}+t^{\text {buff }}\right\} \\
& x_{i \alpha}+x_{j \alpha} \leq 1 \quad \forall(i, j) \in P \forall \alpha \in G
\end{aligned}
$$




\section{FGA BP Constraints}

1. One gate per flight

$$
\sum_{\alpha} x_{i \alpha}=1 \quad \forall i \in F
$$

2. Different gates if standing times of two flights overlap forbidden pairs

$$
\begin{gathered}
P=\left\{(i, j) \in F^{2}: t_{i}^{\text {in }}<t_{j}^{\text {in }}<t_{i}^{\text {out }}+t^{\text {buff }}\right\} \\
x_{i \alpha}+x_{j \alpha} \leq 1 \\
\mathbb{1} \\
x_{i \alpha} \cdot x_{j \alpha}=0
\end{gathered} \quad \forall(i, j) \in P \forall \alpha \in G
$$


www.DLR.de - Chart $7>$ July 13, $2018>$ T. Stollenwerk > Flight Gate Assignment with a Quantum Annealer

\section{QUBO Formulation}

$$
Q(x)=O(x)+\lambda_{\text {one }} C_{\text {one }}(x)+\lambda_{\text {not }} C_{\text {not }}(x)
$$




\section{QUBO Formulation}

$$
Q(x)=O(x)+\lambda_{\text {one }} C_{\text {one }}(x)+\lambda_{\text {not }} C_{\text {not }}(x)
$$

with penalty terms 


\section{QUBO Formulation}

$$
Q(x)=O(x)+\lambda_{\text {one }} C_{\text {one }}(x)+\lambda_{\text {not }} C_{\text {not }}(x)
$$

with penalty terms

$$
C_{\text {one }}(x)=\sum_{i}\left(\sum_{\alpha} x_{i \alpha}-1\right)^{2}
$$




\section{QUBO Formulation}

$$
Q(x)=O(x)+\lambda_{\text {one }} C_{\text {one }}(x)+\lambda_{\text {not }} C_{\text {not }}(x)
$$

with penalty terms

$$
\begin{aligned}
& C_{\text {one }}(x)=\sum_{i}\left(\sum_{\alpha} x_{i \alpha}-1\right)^{2} \\
& C_{\text {not }}(x)=\sum_{\alpha} \sum_{(i, j) \in P} x_{i \alpha} x_{j \alpha}
\end{aligned}
$$




\section{QUBO Formulation}

$$
Q(x)=O(x)+\lambda_{\text {one }} C_{\text {one }}(x)+\lambda_{\text {not }} C_{\text {not }}(x)
$$

with penalty terms

$$
\begin{aligned}
& C_{\text {one }}(x)=\sum_{i}\left(\sum_{\alpha} x_{i \alpha}-1\right)^{2} \\
& C_{\text {not }}(x)=\sum_{\alpha} \sum_{(i, j) \in P} x_{i \alpha} x_{j \alpha}
\end{aligned}
$$

where

$$
C_{\text {one } / \text { not }} \begin{cases}>0, & \text { if constraint is violated } \\ =0, & \text { if constraint is fulfilled }\end{cases}
$$




\section{QUBO Formulation}

$$
Q(x)=O(x)+\lambda_{\text {one }} C_{\text {one }}(x)+\lambda_{\text {not }} C_{\text {not }}(x)
$$

with penalty terms

$$
\begin{aligned}
& C_{\text {one }}(x)=\sum_{i}\left(\sum_{\alpha} x_{i \alpha}-1\right)^{2} \\
& C_{\text {not }}(x)=\sum_{\alpha} \sum_{(i, j) \in P} x_{i \alpha} x_{j \alpha}
\end{aligned}
$$

where

$$
C_{\text {one } / \text { not }} \begin{cases}>0, & \text { if constraint is violated } \\ =0, & \text { if constraint is fulfilled }\end{cases}
$$

Contribution of objective function should not exceed penalty! 


\section{Choice of Penalty Weights}

Need to ensure that a solution always fulfills constraints, hence

$$
\Delta C>\Delta O
$$




\section{Choice of Penalty Weights}

Need to ensure that a solution always fulfills constraints, hence

$$
\Delta C>\Delta O
$$

Comparing coefficients in worst cases for 


\section{Choice of Penalty Weights}

Need to ensure that a solution always fulfills constraints, hence

$$
\Delta C>\Delta O
$$

Comparing coefficients in worst cases for

- not assigning a flight to any gate

$$
\lambda_{\text {one }}>\max _{i, \alpha}\left(n_{i}^{\text {dep }} t_{\alpha}^{\text {dep }}+n_{i}^{\text {arr }} t_{\alpha}^{\text {arr }}+\max _{\beta} T_{\alpha \beta} \sum_{j} N_{i j}\right)
$$




\section{Choice of Penalty Weights}

Need to ensure that a solution always fulfills constraints, hence

$$
\Delta C>\Delta O
$$

Comparing coefficients in worst cases for

- not assigning a flight to any gate

$$
\lambda_{\text {one }}>\max _{i, \alpha}\left(n_{i}^{\text {dep }} t_{\alpha}^{\text {dep }}+n_{i}^{\text {arr }} t_{\alpha}^{\text {arr }}+\max _{\beta} T_{\alpha \beta} \sum_{j} N_{i j}\right)
$$

- assigning a pair of forbidden flights to the same gate

$$
\begin{aligned}
\lambda_{\text {not }}>\max _{i, \alpha, \gamma}\left(\left(n_{i}^{\text {dep }} t_{\alpha}^{\text {dep }}-n_{i}^{\text {dep }} t_{\gamma}^{\text {dep }}\right)\right. & +\left(n_{i}^{\text {arr }} t_{\alpha}^{\text {arr }}-n_{i}^{\text {arr }} t_{\gamma}^{\text {arr }}\right) \\
& \left.+\max _{\beta}\left(T_{\alpha \beta}-T_{\gamma \beta}\right) \sum_{j} N_{i j}\right)
\end{aligned}
$$




\section{Airport Data}

- Flight schedule for one day from a mid-sized European airport 


\section{Airport Data}

- Flight schedule for one day from a mid-sized European airport

- Passenger flow from agent-based simulation of Martin Jung

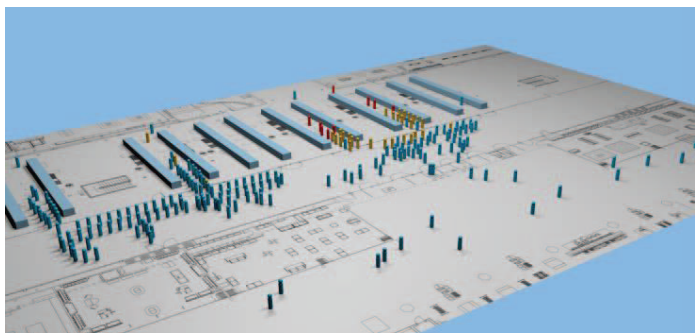

Simulating a multi-airport region to foster individual door-to-door travel, M. Jung et al. 


\section{Airport Data}

- Flight schedule for one day from a mid-sized European airport

- Passenger flow from agent-based simulation of Martin Jung

- Extracted total instance: 293 flights and 97 gates

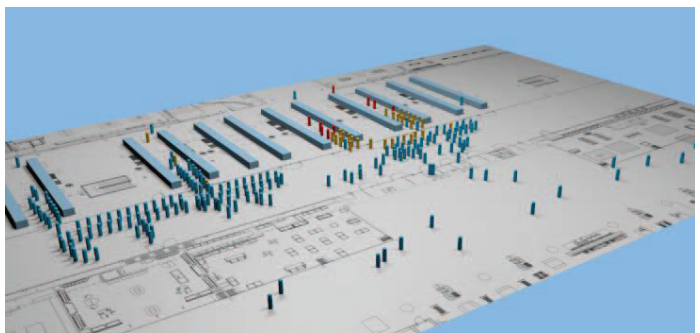

Simulating a multi-airport region to foster individual door-to-door travel, M. Jung et al. 


\section{Airport Data}

- Flight schedule for one day from a mid-sized European airport

- Passenger flow from agent-based simulation of Martin Jung

- Extracted total instance: 293 flights and 97 gates

$\Rightarrow$ Over 28000 binary variables with about 400 Mio. couplings

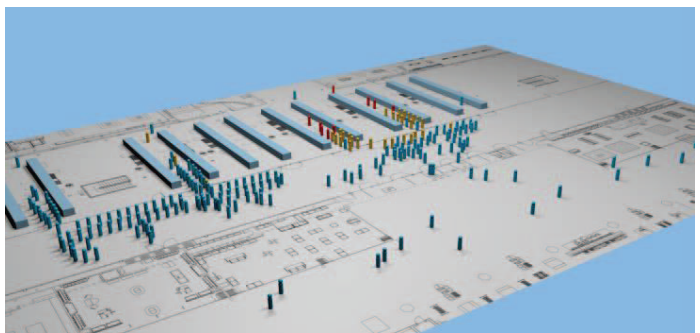

Simulating a multi-airport region to foster individual door-to-door travel, M. Jung et al. 


\section{Instance Preprocessing}

- Removing corrupted data

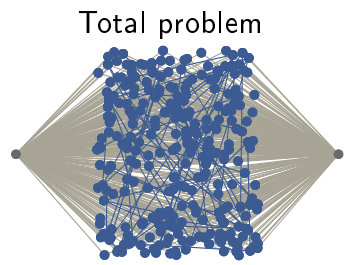

Transfer Passengers
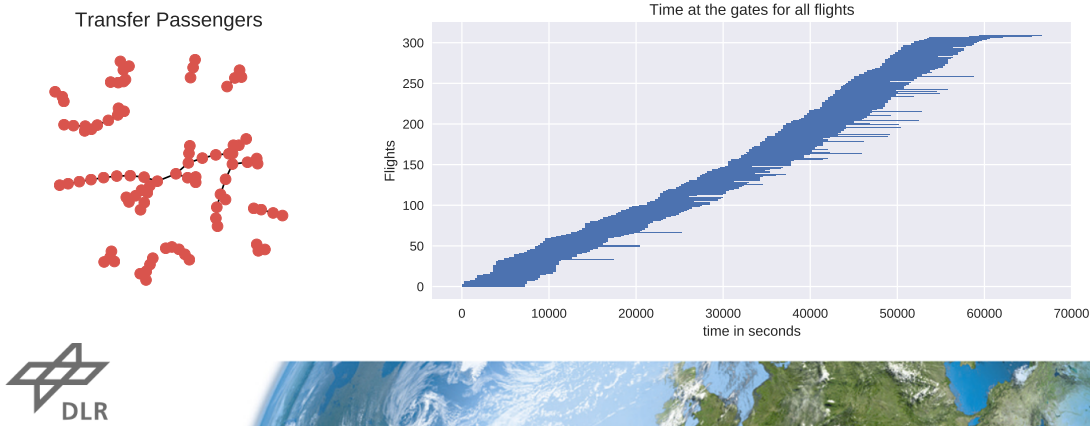


\section{Instance Preprocessing}

- Removing corrupted data

- Splitting too long on-block times

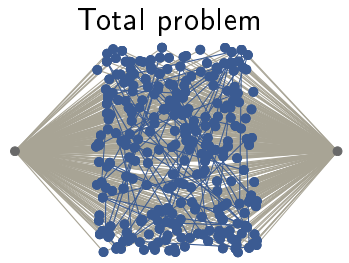

Transfer Passengers
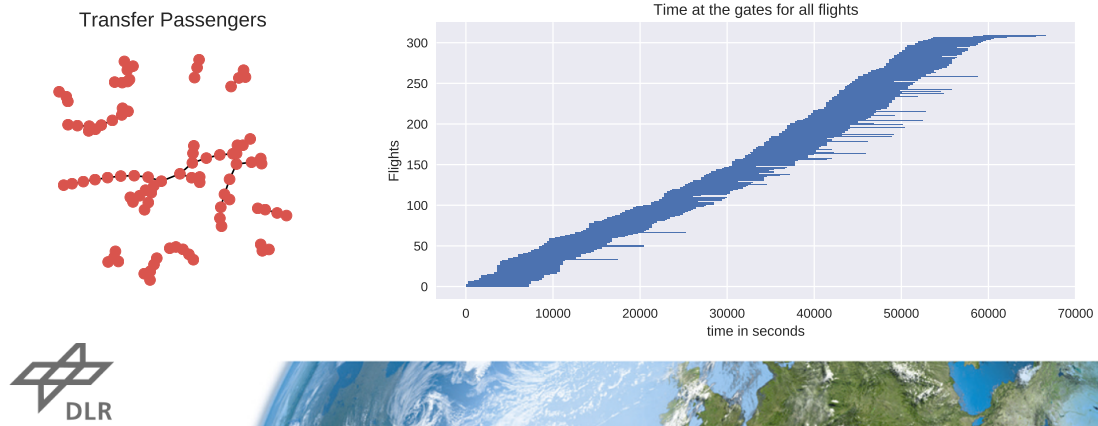


\section{Instance Preprocessing}

- Removing corrupted data

- Splitting too long on-block times

- Reducing to flights with transfers only

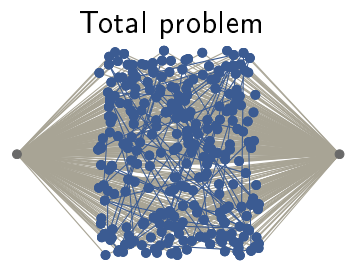

Transfer Passengers
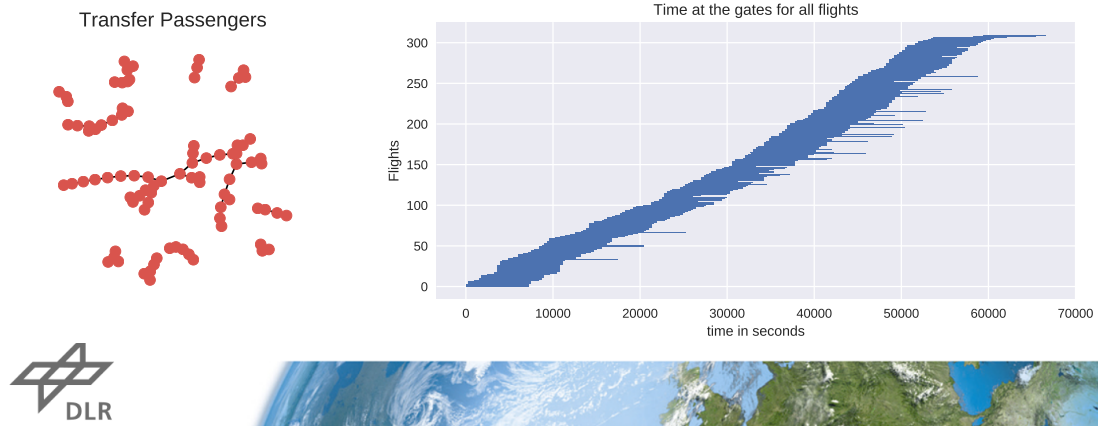


\section{Instance Preprocessing}

- Removing corrupted data

- Splitting too long on-block times

- Reducing to flights with transfers only

$\Rightarrow 89$ remaining flights with 80 transfers

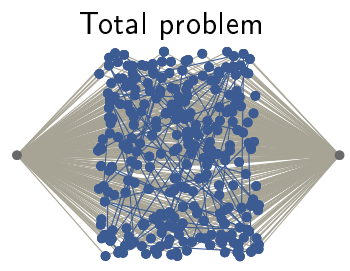

Transfer Passengers

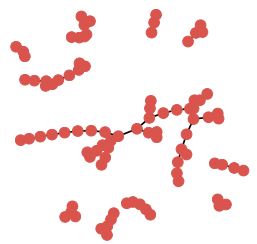

Time at the gates for all flights

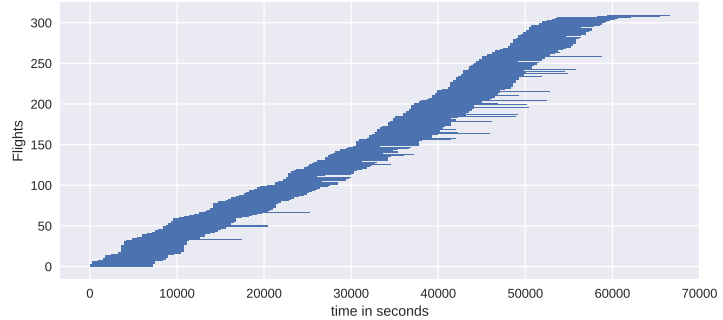




\section{Instance Preprocessing}

- Removing corrupted data

- Splitting too long on-block times

- Reducing to flights with transfers only

$\Rightarrow 89$ remaining flights with 80 transfers

$\Rightarrow$ more than 35 gates

\section{Total problem}

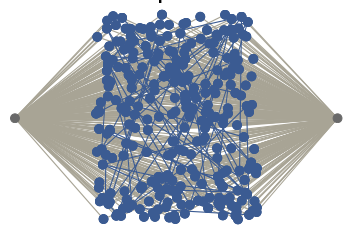

Transfer Passengers
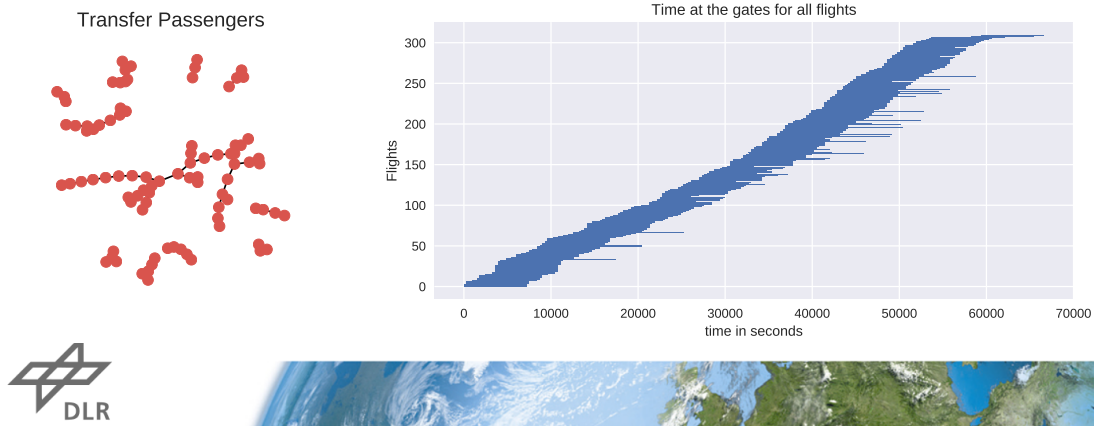
www.DLR.de - Chart $11>$ July 13, $2018>$ T. Stollenwerk > Flight Gate Assignment with a Quantum Annealer

\section{Instance Reduction}

- Slicing by time intervals

Transter Passengers

Forbidden Flight Pairs

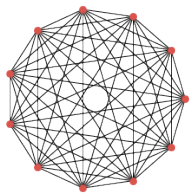

Time at the gates for all flights
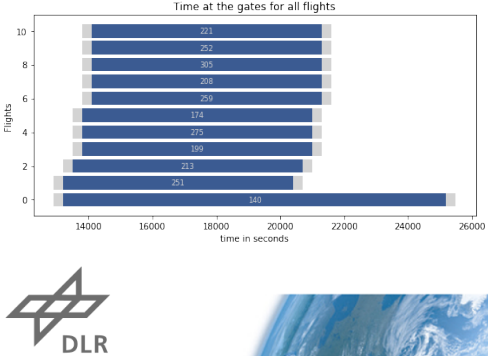


\section{Instance Reduction}

- Slicing by time intervals

Transter Passengers

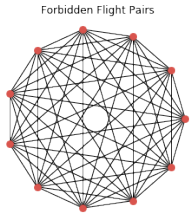

Time at the gates for all flight

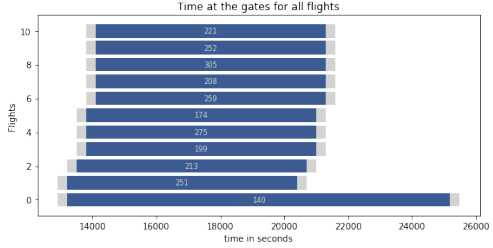

- Connected components of transfer passenger graph
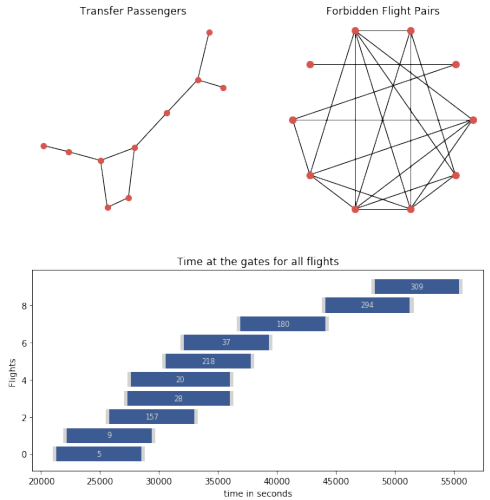

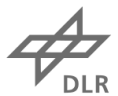




\section{Instance Reduction}

Transfer Passengers

- Use connected components of transfer passenger graph to find small hard instances
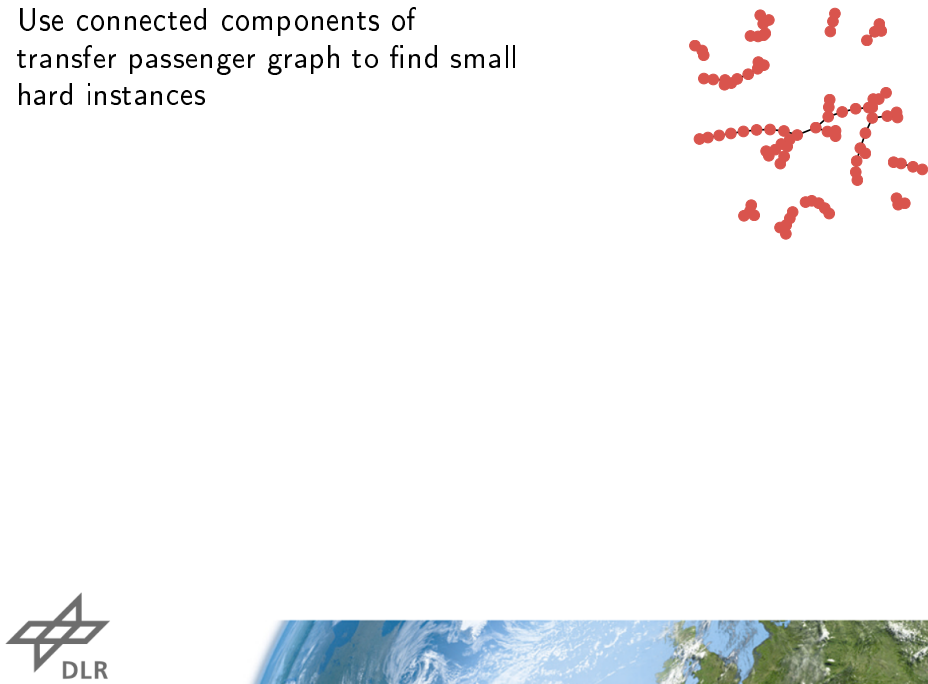


\section{Instance Reduction}

Transfer Passengers

- Use connected components of transfer passenger graph to find small hard instances

- Choose number of gates close number of maximal clique
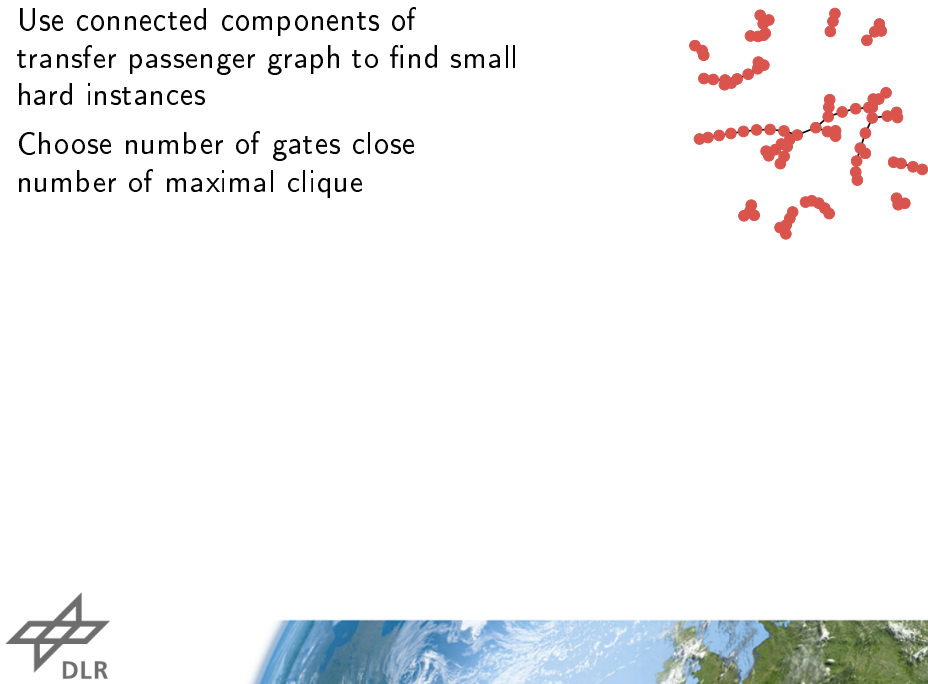


\section{Instance Reduction}

Transfer Passengers

- Use connected components of transfer passenger graph to find small hard instances

- Choose number of gates close number of maximal clique

- Split largest connected component of the transfer passenger graph
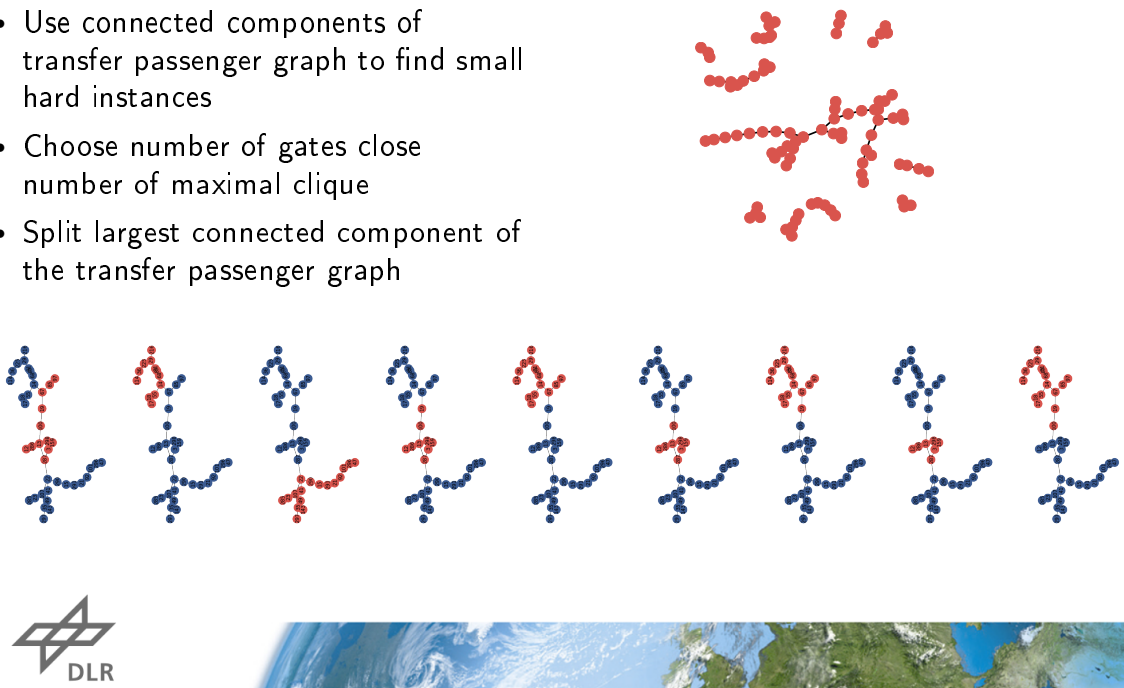


\section{Classical Solution}

- Getting exact solutions using classical solver SCIP
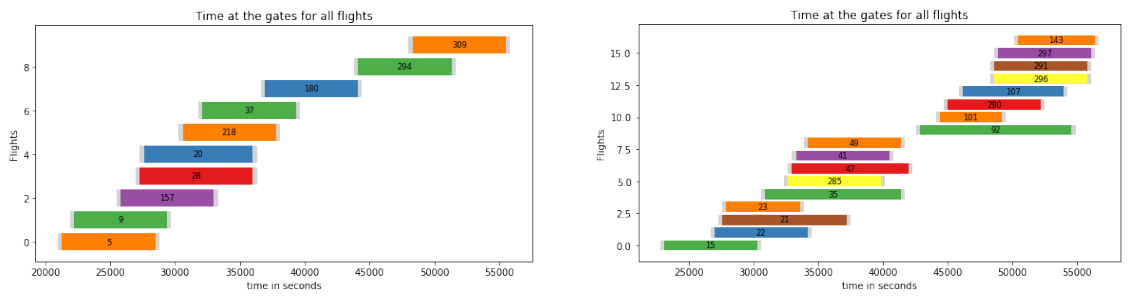


\section{Embedding}

- Standard D-Wave API 


\section{Embedding}

- Standard D-Wave API

- 5 embeddings for each instance

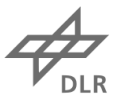




\section{Embedding}

- Standard D-Wave API

- 5 embeddings for each instance

- Quadratic overhead

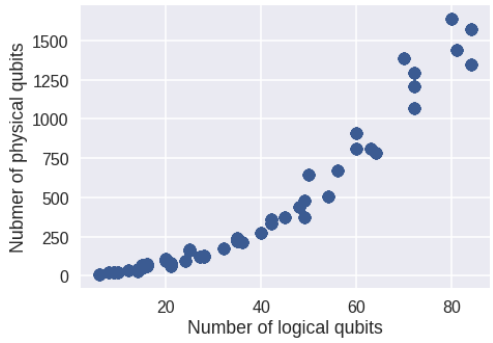




\section{Embedding}

- Standard D-Wave API

- 5 embeddings for each instance

- Quadratic overhead

- Maximum 90 logical qubits

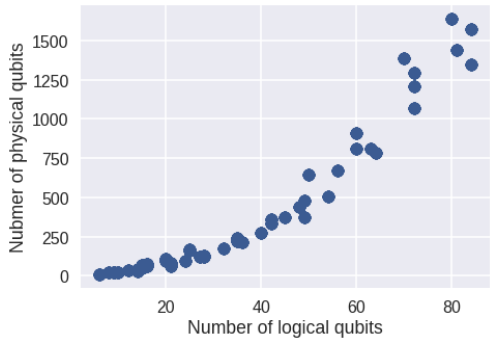




\section{Embedding}

- Standard D-Wave API

- 5 embeddings for each instance

- Quadratic overhead

- Maximum 90 logical qubits

(\#Variables = \#Flights \#Gates $)$

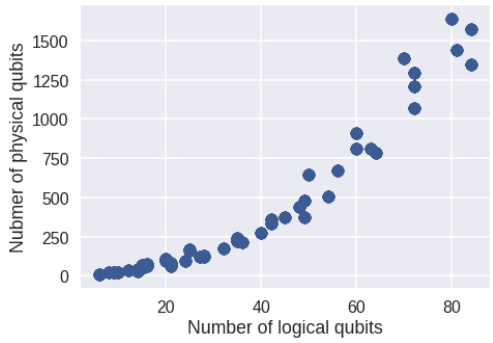




\section{Annealing Results}

- 10000 runs with different parameter settings 


\section{Annealing Results}

- 10000 runs with different parameter settings

- Even for the small instances:

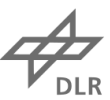




\section{Annealing Results}

- 10000 runs with different parameter settings

- Even for the small instances:

Ratio of maximal to minimal coefficient too large!

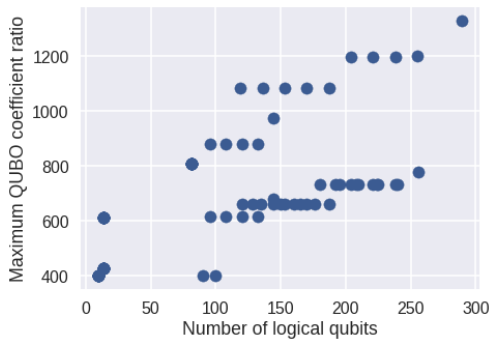




\section{Annealing Results}

- 10000 runs with different parameter settings

- Even for the small instances:

Ratio of maximal to minimal coefficient too large!

$\Rightarrow$ Success probability close to 0

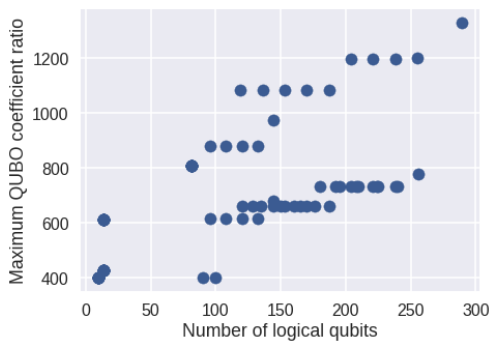




\section{Annealing Results}

- Running structurally equivalent but random instances with much smaller ratios 


\section{Annealing Results}

- Running structurally equivalent but random instances with much smaller ratios

- 10000 runs with different parameter settings 


\section{Annealing Results}

- Running structurally equivalent but random instances with much smaller ratios

- 10000 runs with different parameter settings

- Differentiated results 


\section{Annealing Results}

- Running structurally equivalent but random instances with much smaller ratios

- 10000 runs with different parameter settings

- Differentiated results

- ratio needs to be $<30$

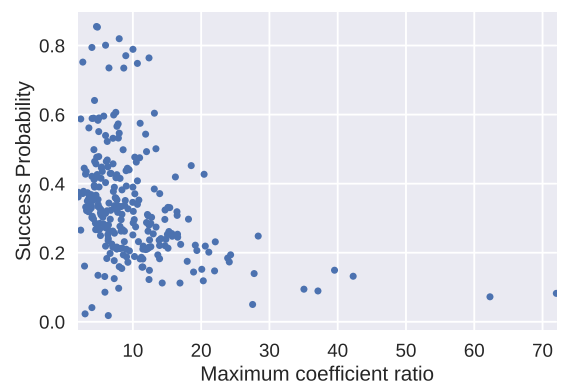




\section{Annealing Results}

- Running structurally equivalent but random instances with much smaller ratios

- 10000 runs with different parameter settings

- Differentiated results

- ratio needs to be $<30$

- wide distribution

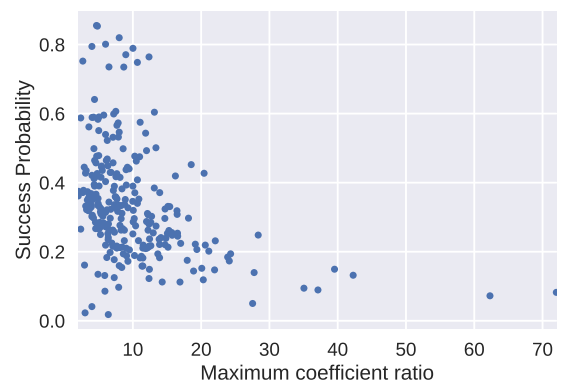




\section{Annealing Results}

- Running structurally equivalent but random instances with much smaller ratios

- 10000 runs with different parameter settings

- Differentiated results

- ratio needs to be $<30$

- wide distribution

- due to other parameters like intra chain coupling of embedding

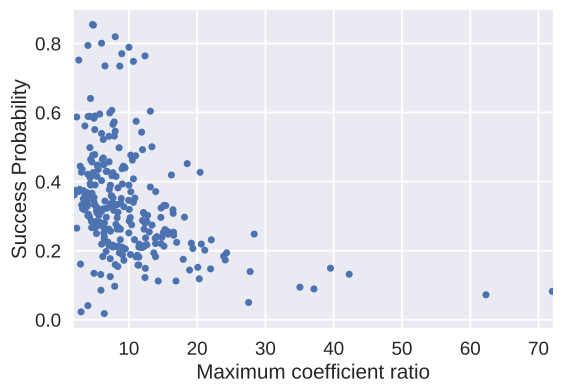


www.DLR.de - Chart $17>$ July 13, $2018>$ T. Stollenwerk > Flight Gate Assignment with a Quantum Annealer

\section{Outlook}

- Adjust embedding/annealing parameters for more clear results

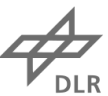


www.DLR.de - Chart $17>$ July 13, $2018>$ T. Stollenwerk > Flight Gate Assignment with a Quantum Annealer

\section{Outlook}

- Adjust embedding/annealing parameters for more clear results

- Investigate new D-Wave features 


\section{Outlook}

- Adjust embedding/annealing parameters for more clear results

- Investigate new D-Wave features

- Using hybrid approach to incorporate classical results 


\section{Outlook}

- Adjust embedding/annealing parameters for more clear results

- Investigate new D-Wave features

- Using hybrid approach to incorporate classical results

- Using QAOA algorithm (related gate based approach) 
www.DLR.de - Chart $18>$ July 13, $2018>$ T. Stollenwerk > Flight Gate Assignment with a Quantum Annealer

\section{Thank You}

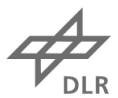

